Notfall Rettungsmed 2017 $\cdot 20: 565-566$ https://doi.org/10.1007/s10049-017-0369-4 Online publiziert: 23. Oktober 2017

๑) Springer Medizin Verlag GmbH 2017

CrossMark

\author{
P. Herrmann ${ }^{1} \cdot$ C. Waydhas ${ }^{2} \cdot$ C. Wrede ${ }^{3} \cdot$ B. W. Böttiger ${ }^{4}$ \\ ${ }^{1}$ Springer Medizin Verlag GmbH, Heidelberg, Deutschland \\ ${ }^{2}$ Klinik und Poliklinik für Unfallchirurgie, Universitätsklinikum Essen, Essen, Deutschland \\ ${ }^{3}$ Notfallzentrum mit Rettungsstelle, HELIOS Klinikum Berlin-Buch, Berlin, Deutschland \\ ${ }^{4}$ Klinik für Anästhesiologie und Operative Intensivmedizin, Universitätsklinikum Köln (AöR), Köln, \\ Deutschland
}

\title{
Zwanzig Jahre Notfall + Rettungsmedizin
}

\section{Kräftig, charmant und langsam erwachsen}

„Die Zeit geht hin, und der Mensch gewahrt es nicht." Dante Alighieri.

Die Gründung der Zeitschrift Notfall + Rettungsmedizin vor nunmehr 20 Jahren (der damals Beteiligte und Erstautor dieser Zeilen hier wundert und freut sich sehr) geschah nicht ohne konkreten Anlass.

Im Jahr 1997 wurde im Sozialgesetzbuch V die Stellung des Notarztes im Rettungsdienst bei der Versorgunglebensbedrohlicher Zustände besonders hervorgehoben. Für diese Versorgungsqualifikation gab es damals im Zeitschriftenbereich in Deutschland ein echtes Vakuum - oder aus Sicht des Verlegers eine echte Chance, ein bislang ungedeckter Bedarf nach qualifizierter Fortbildung und deutschsprachiger Wissenschaft in der Notfallmedizin. Das war der Geburtsgedanke der neuen wissenschaftlich orientierten Zeitschrift Notfall + Rettungsmedizin, deren sog. 0-Nummer entsprechend Ende 1997 erschien (• Abb. 1).

\section{》) Die Druckauflage stieg kontinuierlich auf heute $>10.000$ Exemplare}

Bevor jedoch die erste Ausgabe auf dem Tisch lag, bedurfte es einer nicht unbeträchtlichen Vorarbeit. Mit den Herren Professoren Dick, Lemburg und Schuster fand sich eine exzellente interdisziplinäre Schriftleitung, die angesichts eines überzeugenden Konzepts, eines guten Netzwerks und psychologischen Ge- schicks keine großen Schwierigkeiten hatte, die hellsten Köpfe der Notfallmedizin in Deutschland und Österreich für diese Idee zu begeistern und in das Board der Zeitschrift zu holen.

Prägende Persönlichkeiten der allerersten Stunde waren neben der Schriftleitung die Herausgeber Dr. Dr. Dirks und Prof. Dr. Kreimeier (beide später Schriftleiter von 2008 bis 2013 bzw. 2014) sowie der viel zu früh verstorbene Herr Prof. Dr. Moecke und Herr Prof. Dr. Arntz (später Schriftleiter von 2008 bis 2014).

Die konstituierenden Sitzungen waren geprägt von einer echten Aufbruchsstimmung und der Überzeugung, etwas bewegen zu können. Und tatsächlich: Wo ein Bedarf ist und ein Produkt, das diesen Bedarf deckt und die Zielgruppe auf der richtigen Flughöhe abholt, da entwickelte sich auch Akzeptanz - bald war Notfall + Rettungsmedizin in der notfallmedizinischen Community nicht mehr wegzudenken. Eine kontinuierliche und nachhaltige Steigerung der Auflage von $>10.000$ Exemplaren heute unterstreicht dies in besonderer Weise (• Abb. 2).

Immer wieder hat es die Zeitschrift geschafft, publikatorische Highlights zu setzen. So war ein wichtiger Meilenstein z. B. die erstmalige vollständige Publikation der Lebensrettenden Sofortmaßnahmen des European Resuscitation Council (ERC) auf Deutsch als Sonderausgabe der Notfall + Rettungsmedizin im Jahr 2006. Ab diesem Zeitpunkt wurde dies mit jeder Neuauflage der ERC-Leitlinien wiederholt, in Kooperation mit dem German Resuscitation Council (GRC) opti- miert, und diese Sonderausgaben sind heute eine feststehende vielfach zitierte Größe in der deutschen Notfallmedizin. Und gleichzeitig ist es aus unserer Sicht auch gelungen, den initialen notfall- und rettungsmedizinischen Pioniergeist aufrechtzuerhalten und weiter $\mathrm{zu}$ beflügeln. Bei allen politischen und fachlichen Entwicklungen im notfallmedizinischen Bereich ist unsere Zeitschrift ein Beispiel von gelebter Interdisziplinarität und Interprofessionalität auf hohem Niveau. Alle wesentlichen und in der Notfall- und Rettungsmedizin vertretenen Spieler sind dabei. Die Medizin

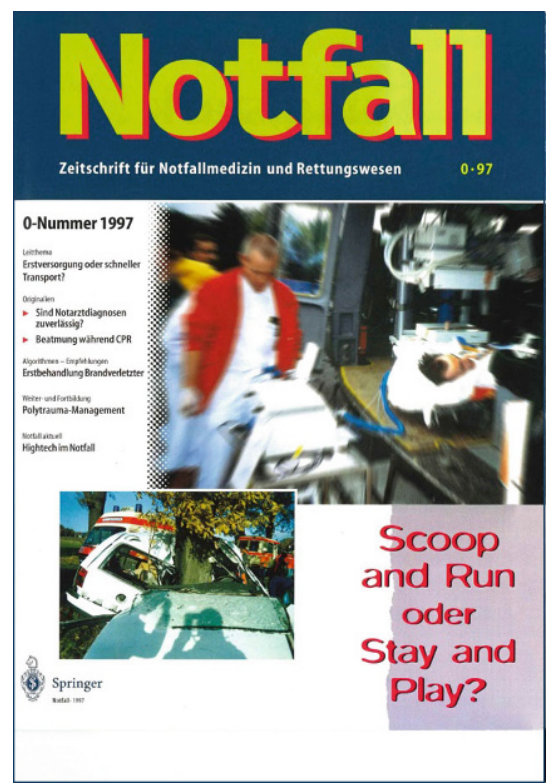

Abb. 1 A Die 0-Nummer aus dem Jahr 1997, damals noch mit dem Titel Notfall. Dieser wurde für Bd. 11998 in Notfall +Rettungsmedizin umbenannt 


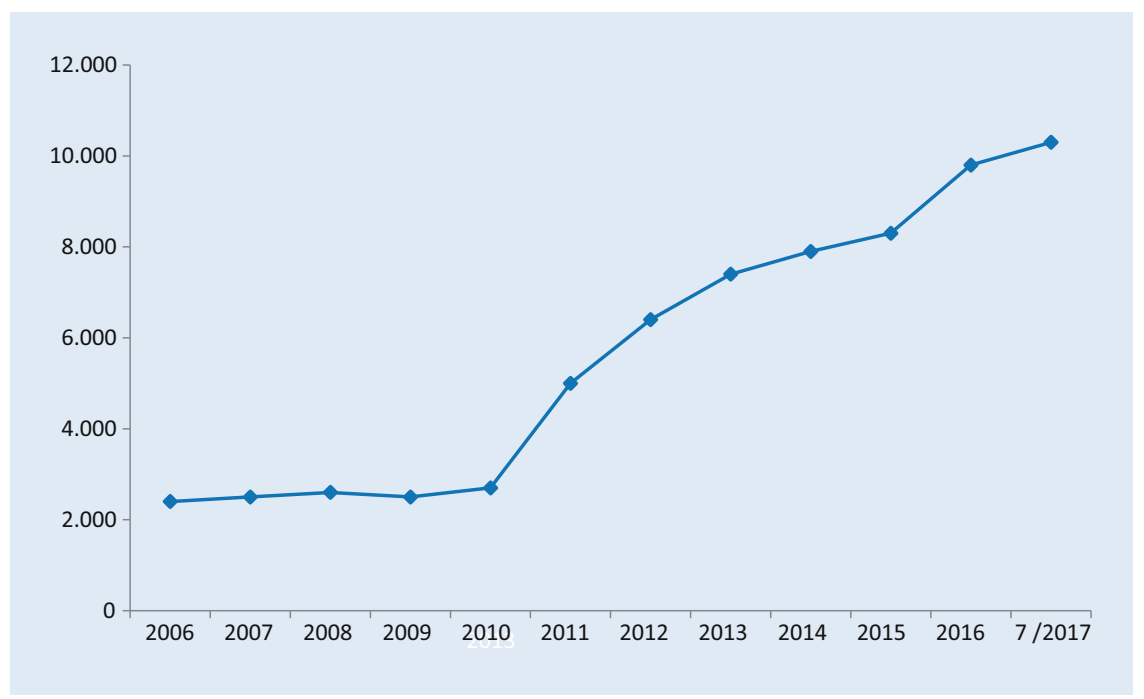

Abb. 2 ॥ Entwicklung der Druckauflage der Zeitschrift Notfall + Rettungsmedizin ab 2006

und der Patient stehen im Mittelpunkt. Wir diskutieren auch politisch, publizieren Leitlinien und Statements auf stets nachhaltige, konstruktive und sachorientierte Art und Weise. Eine fachliche und persönliche Bereicherung für alle Beteiligten, auch für die heutigen Herausgeber und Schriftleiter.

Entsprechend dieser Entwicklung erhielt die Zeitschrift unter der damaligen Leitung von Herrn Professor Kreimeier 2009 einen Impact Factor. Es ist an dieser Stelle einfach bemerkenswert, dass eine deutschsprachige Zeitschrift, die ihren Fokus auch aufFortbildung legt, sich auch auf internationalem Terrain behaupten kann.

Ab dem Jahr 1999 kamen für Notfall + Rettungsmedizin wichtige Organschaften mit Mitgliederbezug hinzu, die eine weitere wesentliche Basis unserer Zeitschrift sind und Ausdruck der hervorragenden Vernetzung und Akzeptanz: Im Jahr 1999 die Deutsche Interdisziplinäre Vereinigung für Intensiv- und Notfallmedizin e. V. (DIVI), 2008 der Deutsche Rat für Wiederbelebung - German Resuscitation Council e.V. (GRC) und der Österreichische Rat für Wiederbelebung - Austrian Resuscitation Council (ARC), 2010 dann die Arbeitsgemeinschaft Südwestdeutscher Notärzte e.V. (agswn) und die Deutsche Gesellschaft Interdisziplinäre Notfall- und Akutmedizin e. V. (DGINA). Ein Jahr später der Deutsche Berufsverband Rettungsdienst

e. V. (DBRD), der mittlerweile einer der größten Fachgruppen in diesem Bereich darstellt und was auch Ausdruck der Interprofessionalität unserer Zeitschrift ist. Und aktuell, seit diesem Jahr ist der Bundesverband Ärztlicher Leiter Rettungsdienst (BV ÄLRD) an Bord. Ein sehr schöner Geburtstagsstrauß, der hier entstanden ist.

\section{Was bewegt uns ganz besonders?}

Ein riesiger Dank gebührt den erstklassigen Experten und besonderen Persönlichkeiten, die sich über die Jahre hinweg mit ihrer Expertise, Organisationstalent und diplomatischem Geschick als Autoren, Gutachter oder Editoren eingebracht haben.

\section{1) Notfall + Rettungsmedizin wird Trends weiterhin frühzeitig erkennen und die richtigen Schwerpunkte setzen}

Diese Zeitschrift ist ein kleiner Kosmos von Menschen mit unterschiedlichen Fähigkeiten und Verantwortlichkeiten, die im Zusammenspiel sehr gut harmonieren und kooperieren. Der Dank gebührt aber selbstverständlich auch Ihnen, unserer treuen Leserschaft, ohne die es diese Zeitschrift nicht geben würde.
Ob der spürbaren und oben skizzierten Entwicklung bleibt die feste Gewissheit, dass unsere Zeitschrift auf einem sehr guten Weg ist. Sie wird inhaltlich auf der Höhe der Zeit bleiben, Trends in der Notfallmedizin frühzeitig erkennen und mit den richtigen Mitteln die richtigen Schwerpunkte setzen. Dafür stehen ein Schriftleitergremium und ein Herausgeberboard, das alle Bereiche der Notfallund Rettungsmedizin in bester Weise repräsentiert. Dabei werden wir uns dem Wandel der Mediennutzung stellen und immer ein offenes Ohr für die Bedürfnisse der NutzerInnen und LeserInnen und Leser haben. Das ist das klare Commitment und Versprechen des Verlags und der Schriftleitung, an dem wir sehr gerne auch in Zukunft gemessen werden wollen.

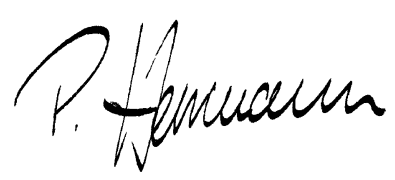

Paul Herrmann

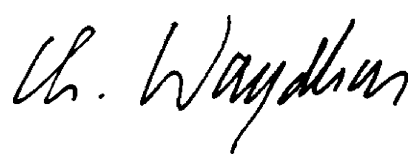

Christian Waydhas

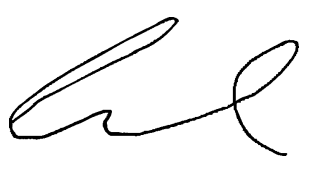

Christian Wrede

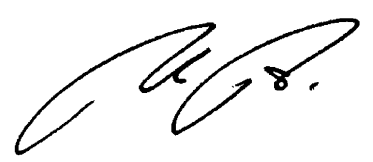

Bernd W. Böttiger

\section{Korrespondenzadresse}

\section{Dr. P. Herrmann}

Springer Medizin Verlag GmbH

Tiergartenstraße 17, 69121, Heidelberg,

Deutschland

notfallundrettungsmedizin@springer.com

Interessenkonflikt. Dr. P. Herrmann ist verlagsseitiger Mitbegründer der Zeitschrift Notfall + Rettungsmedizin; Professor B. W. Böttiger, Professor C. Waydhas und Professor C. Wrede haben die Schriftleitung der Notfall + Rettungsmedizin inne. 